\title{
A NOTE ON AREA VARIABLES IN REGGE CALCULUS
}

\author{
John W. Barrett, Martin Roček, Ruth M. Williams
}

10 October 1997

\begin{abstract}
We consider the possibility of setting up a new version of Regge calculus in four dimensions with areas of triangles as the basic variables rather than the edgelengths. The difficulties and restrictions of this approach are discussed.
\end{abstract}

In three dimensions, the topological invariant for three-manifolds constructed by Turaev and Viro [1] reduces in the semi-classical limit to the expression written down more than twenty years earlier by Ponzano and Regge [2]. In the case of a very fine triangulation of the manifold, Ponzano and Regge had pointed out an intriguing connection between their state sum and the Feynman path integral for three-dimensional quantum gravity using the Regge calculus action [3]. A prerequisite for this was the very obvious one that in the Turaev-Viro and PonzanoRegge expressions, 'colourings' or angular momentum variables are assigned to the edges (links) of the triangulation, and in Regge calculus the edge lengths are indeed the variables playing the role of the metric tensor in the continuum theory.

An extension of the Turaev-Viro type of state sum to four dimensions [4,5] involved assigning the colourings or variables to the two-dimensional faces of the triangulation. The relationship between this work and the loop representation of quantum gravity has been explored by Rovelli [6], who suggested that it should be related to a modified version of Regge calculus in four dimensions, in which the independent variables would be the areas of the triangles rather than the edge lengths. Prompted by this remark, we set out to explore this possibility.

More recently, the idea of the areas of triangles as variables in state sum models of quantum gravity featured in $[7,8]$, and the relation of these models to continuum Lagrangians was explored in $[9,10]$.

Rovelli's ideas fitted in with earlier work by Mäkelä [11] who considered a version of Regge calculus using the areas of triangles as coordinates, based on a modification of the Ashtekar formalism. Mäkelä noted that in Regge calculus, there are constraints among the area variables, and concentrated on taking account of these constraints so that his formalism still corresponded to using edge lengths [12]. In

Preprint no. ITP-SB-97-59 
contrast, in the present work our intention is to explore the possibility of treating the areas as the fundamental and independent variables.

The fact that a four-simplex has the same number of triangular faces as edges is very suggestive, and as Rovelli claims, one would expect to be able to invert the relationship between $A_{t}$, the area of triangle $t$, and the squares of the edge-lengths, $s_{i}$, in order to express the Regge action

$$
I\left(s_{i}\right)=\sum_{t} A_{t}\left(s_{i}\right) \epsilon_{t}\left(s_{i}\right)
$$

in terms of the areas $A_{t}$. Here $\epsilon_{t}$ is the deficit angle at triangle t. Then one could look for stationary points of the action, leading to discrete solutions of Einstein's equations. However, as we shall see, there are a number of difficulties with this approach.

Firstly let us look at the problem of inverting the relationship between lengths and areas for a given four-simplex. Label the vertices $1,2, \ldots, 5$; let $s_{i j}$ be the length-squared of the edge joining vertices $i$ and $j$ and $A_{i j k}$ the area of the triangle with vertices $i, j, k$. Then

$$
A_{i j k}^{2}=\left(2\left(s_{i j} s_{j k}+s_{i k} s_{j k}+s_{i j} s_{i k}\right)-s_{i j}^{2}-s_{j k}^{2}-s_{i k}^{2}\right) / 16 .
$$

As Philip Tuckey pointed out, there are pairs of four-simplexes, with squared edge-lengths $(1,1,1,1,1,1,1,1,1, a)$ which have the same triangle areas, but with different values of $a$ either side of 2 [13]. This follows from the fact that the $A_{i j k}^{2}$ are quadratic in $a$ and are all a maximum at $a=2$. This corresponds to a geometry in which some of the triangles are right-angled. Thus there would have to be a restriction on the class of edge-lengths considered, corresponding to a restriction on the class of metrics, to avoid this two-fold ambiguity. This can be done by restricting to some neighbourhood of the regular four-simplex.

There is another problem in going from edge-length variables to areas. Although there is a match between the numbers of these for a single four-simplex, this does not hold for a collection of four-simplexes joined together. For example, consider two four-simplexes meeting on a common tetrahedral face. This complex has 14 edges but 16 triangles. For any closed four-manifold, the number of triangles is greater than or equal to $4 / 3$ times the number of edges. Thus even if the edgelengths are restricted so that the transformation to the set of areas is injective, there will be some complicated constraint equations among the areas which arise in this way.

To explore this problem further, consider two four-simplexes as separate entities, each with the areas of its ten faces specified. Then, assuming a restriction to geometries where the transformation between areas and edge lengths is 1-1, we solve for the edge lengths of the two four-simplexes. Now return to the situation where the two four-simplexes are joined on a common tetrahedral face and require that the areas of the common triangles be equal. Since a tetrahedron has six edges and four triangles, the areas certainly do not determine the edge lengths of the tetrahedron uniquely, so we can envisage a bizarre situation where the edge lengths 
of the common tetrahedron have different values, depending on which four-simplex they are considered to be part of.

For example, suppose that for one four-simplex, all the squared areas are 1, and the squared edge lengths are all $4 / \sqrt{3}$. Another four-simplex shares a tetrahedron, so four of its squared areas must be 1. Suppose that of the remaining six, two more are 1 , but the four others are $625 / 768$. The solution for the squared edge lengths in this four-simplex is that two opposite sides of the common tetrahedron have squared-length 3, while the remaining eight edges have squared-length 25/12. (The Jacobian of the transformation is non-zero for both four-simplexes). We see the impossibility of interpreting the edge lengths as real physical quantities in the usual sense. However since the edge lengths are well-defined within each simplex, it is still possible to define the metric in terms of them in the usual way within each simplex.

Finally we need to see what types of discrete solutions of Einstein's equations we obtain, for the restricted class of metrics avoiding the 2-fold ambiguity. Then, the deficit angles are determined unambiguously by the areas, since each 4 -simplex has well-determined hyperdihedral angles. The action is then

$$
I\left(A_{s}\right)=\sum_{t} A_{t} \epsilon_{t}\left(A_{s}\right)
$$

where the sum is over all triangles $t$. Variation with respect to $A_{u}$ gives

$$
0=\epsilon_{u}\left(A_{s}\right)+\sum_{t} A_{t} \frac{\partial \epsilon_{t}}{\partial A_{u}}=\epsilon_{u}\left(A_{s}\right)+\sum_{t} A_{t} \frac{\partial}{\partial A_{u}}\left(2 \pi-\sum_{j} \theta_{t}^{j}\right)
$$

where $\theta_{t}^{j}$ is the internal hyperdihedral angle at triangle $\mathrm{t}$ in four-simplex $\mathrm{j}$, and the second sum is over those $j$ containing $t$.

Using the chain rule and interchanging the order of summation over triangles and over four-simplices, we obtain

$$
0=\epsilon_{u}\left(A_{s}\right)-\sum_{j} \sum_{t} A_{t} \sum_{i} \frac{\partial \theta_{t}^{j}}{\partial s_{i}} \frac{\partial s_{i}}{\partial A_{u}}
$$

where $t$ now runs over the triangles in 4 -simplex $j$, and $i$ runs over the edges for $j$, with $s_{i}$ the length squared of $i$.

Now Regge proved [3] that for the triangles in a four-simplex,

$$
\sum_{t} A_{t} \frac{\partial \theta_{t}^{j}}{\partial s_{i}}=0
$$

and then, since the Jacobian $\partial s_{i} / \partial A_{u}$ is non-singular, the variational equation reduces to $\epsilon_{u}=0$ for all $u$. In Regge calculus, this condition would mean the space is locally flat. However in our theory the deficit angle $\epsilon$ does not measure the holonomy of a connection and so there is no such simple conclusion to be drawn. 


\section{Conclusions}

Making the areas of triangles independent variables for Regge calculus is possible, but the price for this is that there appears to be no well-defined metric geometry. Work is continuing on this puzzling aspect of the formalism. The action can still be defined, as the dihedral angles are determined in each simplex, and these are all that are required to define the deficit angles. A calculation shows that the variational equations imply that these deficit angles are all zero, but this does not imply that the solutions are flat metrics.

\section{ACKNOWLEDGEMENTS}

We are grateful to Francis Archer and Philip Tuckey for helpful discussions. Martin Roček was supported by NSF grant no. PHY9722101.

\section{REFERENCES}

1. V.G. Turaev and O.Y. Viro, State sum invariants of 3-Manifolds and quantum 6j Symbols, Topology 31 (1992), 865-902.

2. G. Ponzano and T. Regge, Semiclassical limit of Racah coefficients, Spectroscopic and group theoretical methods in Physics (F. Bloch, ed.), North-Holland, 1968, pp. 1-58.

3. T. Regge, General relativity without coordinates, Nuovo Cimento 19 (1961), 558-571.

4. H. Ooguri, Topological Lattice Models in Four Dimensions, Mod. Phys. Lett. A7 (1992), 2799-2810.

5. L. Crane, L. Kauffman and D. Yetter, State Sum invariants of 4-Manifolds, J. Knot Theory Ram. 6 (1997), 177-234.

6. C. Rovelli, The basis of the Ponzano-Regge-Turaev-Viro-Ooguri model is the loop representation basis, Phys. Rev. D48 (1993), 2702-2707.

7. J.W. Barrett, L. Crane, Relativistic spin networks and quantum gravity, J. Math. Phys. 39 (1998), 3296-3302.

8. J.C. Baez, Spin foam models, Class. Quant. Grav. 15 (1998), 1827-1858.

9. M.P. Reisenberger, Classical Euclidean general relativity from "left-handed area = righthanded area", gr-qc/9804061.

10. R. De Pietri, L. Freidel, so(4) Plebanski Action and Relativistic Spin Foam Model, grqc/9804071.

11. J. Mäkelä, Phase space coordinates and the Hamiltonian constraint of Regge calculus, Phys. Rev. D49 (1994), 2882-2896.

12. J. Mäkelä, Variation of Area Variables in Regge Calculus, gr-qc/9801022.

13. J.W. Barrett, First order Regge calculus, Class. Quant. Grav. 11 (1994), 2723-2730.

Department of Mathematics, University of Nottingham, University Park, NotTINGHAM, NG7 2RD, UK

E-mail address: jwb@maths.nott.ac.uk

Institute for Theoretical Physics State University of New York at Stony Brook STONY BRoOK NY 11794-3840 USA

E-mail address: rocek@insti.physics.sunysb.edu

DAMTP, Silver Street, Cambridge CB3 9EW, UK

E-mail address: rmw7@damtp.cam.ac.uk 